\title{
A HIGH RESOLUTION IMAGING DETECTOR FOR TeV GAMMA-RAY ASTRONOMY
}

M.F. CAWLEY 1 , D.J. FEGAN 2 , K. HARRIS 3 , A.M. HILLAS 4 , P.W. KWOK 3 R.C. LAMB ${ }^{5}$, M.J. LANG ${ }^{2}$, D.A. LEWIS 5 , D. MACOMB 5 , P.T. REYNOLDS ${ }^{2}$, D.J. SCHMID ${ }^{5 *}$, G. VACANTI ${ }^{5}$, T.C. WEEKES ${ }^{3}$.

1. Physics Department, St. Patrick's College, Maynooth, Co. Kildare, Ireland.

2. Physics Department, University College, Belfield, Dublin 4, Ireland.

3. Harvard-Smithsonian Center for Astrophysics, Whipple Observatory, Amado, $A Z$ 85645-0097.

4. Physics Department, University of Leeds, Leeds LS2 9JT, U.K.

5. Physics Department, Iowa State University, Ames, IA 50011.

* Present address: Physics Department, University of Minnesota, Minneapolis, MN 55455.

(Received 5 September, 1989; accepted 20 December, 1989)

\begin{abstract}
Details are presented of an atmospheric Cherenkov telescope for use in very high energy gamma-ray astronomy which consists of a cluster of 109 close-packed pliotomultiplier tubes at the focus of a 10 meter optical reflector. The images of the Cherenkov flashes generated both by gamma-ray and charged cosmic-ray events are digitized and recorded. Subsequent off-line analysis of the images improves the significance of the signal to noise ratio by a factor of 10 compared with nonimaging techniques.
\end{abstract}

\section{Introduction}

It is convenient to subdivide gamma-ray astronomy into several energy regions, each region utilizing distinct experimental techniques. The Earth's atmosphere is opaque to gamma radiation and detectors for energies less than $10 \mathrm{GeV}$ must be carried to high altitude by satellites or balloons. At high mountain altitudes, above $0.1 \mathrm{PeV}$, the air shower initiated by the primary gamma ray may be detected by an array of particle detectors operating in coincidence. Neither of these techniques is feasible for the intermediate energy region; fluxes are too low for satellite detectors, and the secondary particles in the cascades are virtually all absorbed in the atmosphere before reaching ground level. The cascades may, however, be detected via the Cherenkov light generated by the passage of the charged secondaries through the air. This light, peaking in the far blue and near UV spectral regions, acts as a penetrating component of the cascade, and may be detected using large optical collectors in conjunction with fast phototubes and electronics at a dark site. Using the atmospheric Cherenkov technique, primary gamma rays with energies in excess of $0.1 \mathrm{TeV}$ may be detected, thus filling much of the gap between the satellite and particle detector regions.

When used in its simplest form, the technique lacks sensitivity due to a high rate of background events induced by the isotropic charged primary cosmic rays. In this paper, details are presented of a detector which overcomes this limitation by using images of the Cherenkov light flash to determine the nature and arrival direction of

Experimental Astronomy 1: 173-193, 1990.

(c) 1990 Kluwer Academic Publishers. Printed in the Netherlands. 
the primary. In brief, an array of closely packed photomultiplier tubes (PMTs) placed in the focal plane of a $10 \mathrm{~m}$ diameter optical reflector is used to record the image of the Cherenkov flash (duration of about $10 \mathrm{nsec}$ ). Off-line analysis is then used to isolate the small gamma-ray component according to perscriptions based on Monte Carlo simulations (Hillas, 1985). This second generation, high-resolution camera is deployed at the Whipple Observatory in southern Arizona (latitude $31^{\circ} 41^{\prime}$, longitude $110^{\circ} 53^{\prime}$, altitude $2320 \mathrm{~m}$ ). Details of an early version of the imaging detector have been presented elsewhere (Fegan et al. 1983; Cawley et al. 1985). The efficacy of the technique using that detector was experimentally verified with the detection of a gamma-ray signal from the Crab Nebula at a level of nine standard deviations above the background (Weekes et al,, 1989). The upgraded version of the imaging system, to be described here, has verified this detection at the $15 \sigma$ level (Lang et al. 1989). These successful detections indicate a sensitivity to gamma rays unrivalled by any other technique presently being used in ground-based gamma-ray astronomy.

\section{Optics}

Since the gamma-ray telescope records images of the Cherenkov light from cosmic-ray or gamma-ray initiated showers, it is necessary that the image-forming optic have sufficient angular resolution over its entire field of view. The characteristic angular size of a $1 \mathrm{TeV}$ gamma-ray image is about $0.5^{\circ}$; Monte Carlo simulations indicate structure on finer scales (Hillas, 1985). The Whipple Observatory $10 \mathrm{~m}$ optical reflector (fig. 1) has an unusual optical design (Rieke, 1969) which reduces classical aberrations while maintaining a large aperture and compact size. As shown below, point spread functions have a FWHM of only $0.12^{\circ}$.

The reflector is a tesselated structure consisting of 248 spherical mirrors, each 61 $\mathrm{cm}$ across, arranged in a hexagonal pattem with nine rings (fig 1a). The mirrors are supported by a tubular steel framework forming them into an overall envelope, a 7.3 meter radius bowl with a 10 meter aperture. The individual mirrors have 14.6 meter radii of curvature and are not aligned to be tangent to the overall structure. Instead they are pointed toward a position along the optic axis at distance 14.6 meters from the reflector. As shown by Davies and Cotton (1957) such a reflector forms a focal surface at the center of curvature of the bowl, 7.3 meters from the mirrors (fig. $1 \mathrm{~b}$ ). One disadvantage of this type of design for gamma-ray astronomy is that rays striking facet mirrors in different rings have different transit times to the focal surface. This introduces an additional time spread into the burst of detected Cherenkov light. For the $10 \mathrm{~m}$ reflector, this additional time width of $6 \mathrm{nsec}$ tends to mask any intrinsic difference in the time profiles of gamma-ray-initiated and proton-initiated showers.

The reflector has excellent optical properties at the center of the field of view. The only important aberration for a point source at infinity and on the optic axis is astigmatism in individual facet mirrors near the edge of the reflector (Rieke, 1969). As the point source moves off the optic axis and the incoming rays are no longer parallel to it, a form of coma quickly becomes the dominant aberration (Lewis, 1989). Due to the unusual design, the comatic circles in the focal plane arising from rings on the reflector are all tangent to each other at a single point. Consequently for point source images not on the optic axis but still within the $4^{\circ}$ field of view, the FWHM of the images is only slightly larger. However there is significant loss of light from the region near maximum intensity of off-axis images into a surrounding region of 
(a)

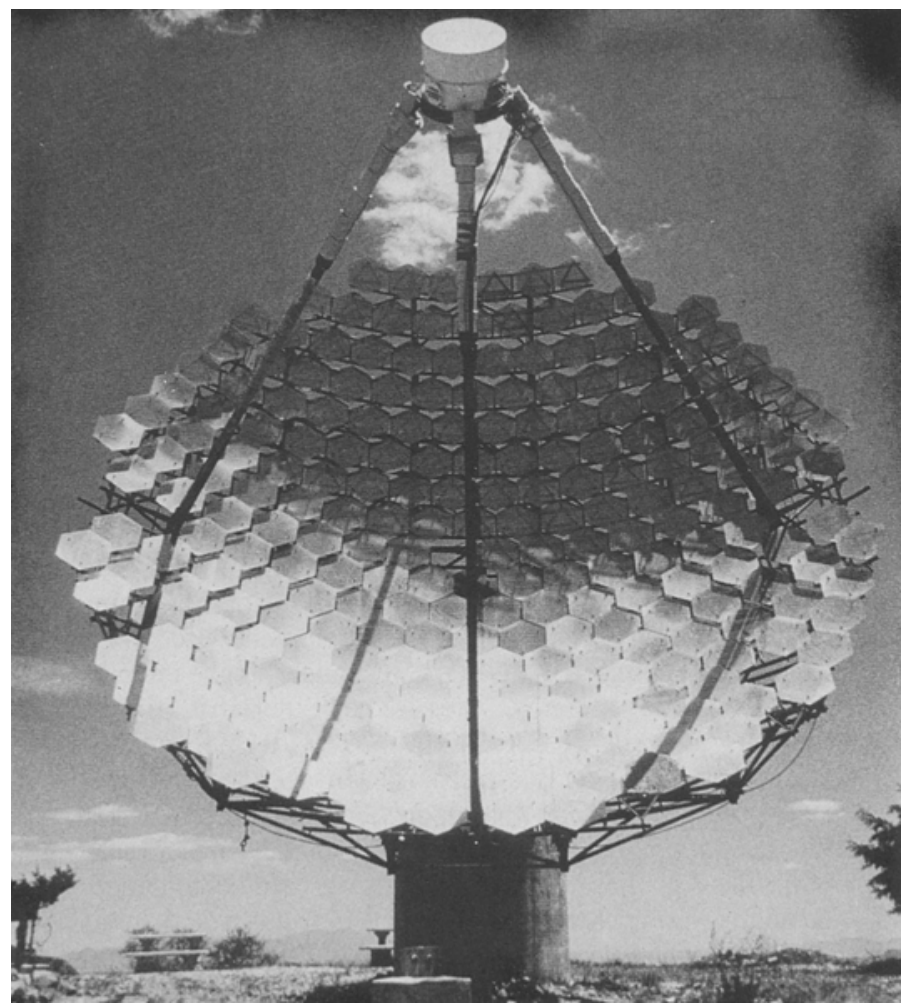

Mirror Located

at angle $\theta$

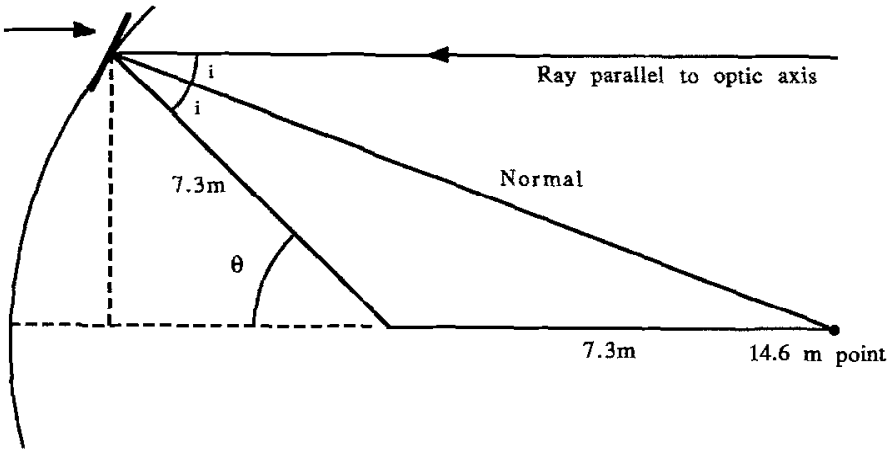

Figure 1. (a) The $10 \mathrm{~m}$ Reflector on Mt. Hopkins, Arizona. (b) The basic geometry of the spherical reflector. A ray parallel to the optic axis strikes the center of a facet mirror displaced from the center of the reflector. The normal from the center of the mirror points towards a point on the optic axis $14.6 \mathrm{~m}$ from the reflector. 
large area but low intensity. The optical properties of the 10 meter reflector have been characterized by ray-tracing calculations both on and off the optic axis and these are incorporated into Monte Carlo simulations of the response of the detector to Cherenkov light from showers (Lewis, 1989).

Measured point spread functions of Polaris are shown in fig. 2 with the star in the center of the field of view and with the star $1.25^{\circ}$ from the center. The profile at $1.25^{\circ}$ from the center was measured radially and the effect of coma is apparent in the asymmetric lineshape with a tail pointing away from the optic axis. The FWHM is approximately $0.12^{\circ}$ for the profile measured at the center and $0.14^{\circ}$ for the off-axis profile.

\section{The Camera Focal Plane Detector}

The basic detector consists of 91 close-packed PMTs of diameter $2.9 \mathrm{~cm}$ arranged in a hexagonal pattern (fig. 3). These are surrounded by an outer ring of 18 PMTs of 5.0 $\mathrm{cm}$ diameter to give a total of 109 pixels. The size of the PMTs is dictated by the desire to extract the maximum angular resolution taking into account the measured optical aberrations of the $10 \mathrm{~m}$ rellector. The basic element used is the Hamamatsu phototube, R1398 which was chosen for its fast response, high quantum efficiency in the blue, and large photocathode sensitive area. The outermost ring of PMTs were those used in the previous camera (RCA6342/IV). The R1398 phototubes have UV transmitting glass windows giving increased response in the near ultraviolet. As proton showers have an enhanced ultraviolet component (because of the local muon component), increased sensitivity in the near-ultraviolet is a disadvantage in a non-imaging system. In fact it has been used as a anticoincidence to reject the background events (Grindlay 1971; Stepanian et al. 1983). In an imaging system the increased sensitivity to the muon component is a definite advantage as it permits increased discrimination on the basis of the enlarged shower images that the muon component produces.

The inner 91 PMTs are arranged in five concentric hexagonal "rings" which are designated as zones 0 through 5 (zone 0 is the center tube, zone 5 is the outer ring of 30 tubes, etc.). To minimize dead-space between the PMTs, the electrostatic and magnetic shields are coated on the outside of the PMT. A center to center spacing of $3.2 \mathrm{~cm}$ is therefore possible. Because of the closepacking and large photocathode effective area, the camera is $62.5 \%$ photocathode sensitive in this inner region. The dynode chains on the PMT bases are encapsulated and the signal is taken directly through $50 \mathrm{~m}$ of RG58 coaxial cable to the trigger and processing electronics. Each PMT has an individual high voltage power supply which is used to control the gain. No optical servo lamps are used.

An array of LEDs is used to continuously monitor the 109 PMT currents and therefore the sky brightness. The LEDs are arranged in a hexagonal pattern identical with the array of PMTs, and the brightness of each LED is proportional to the tube current. If an adjustable threshold (usually 40 microamps) is crossed, the color of the LED changes from green to red, indicating to the observer that a star is crossing the tube. The LED display also provides buffered outputs through which the current on any of the PMTs can be read directly with a digital meter. The display has two primary purposes. The first is to monitor stars as they wander through the rotating field of view of the alt-az telescope. Bright stars $\left(m_{v}<3\right)$ introduce additional night 


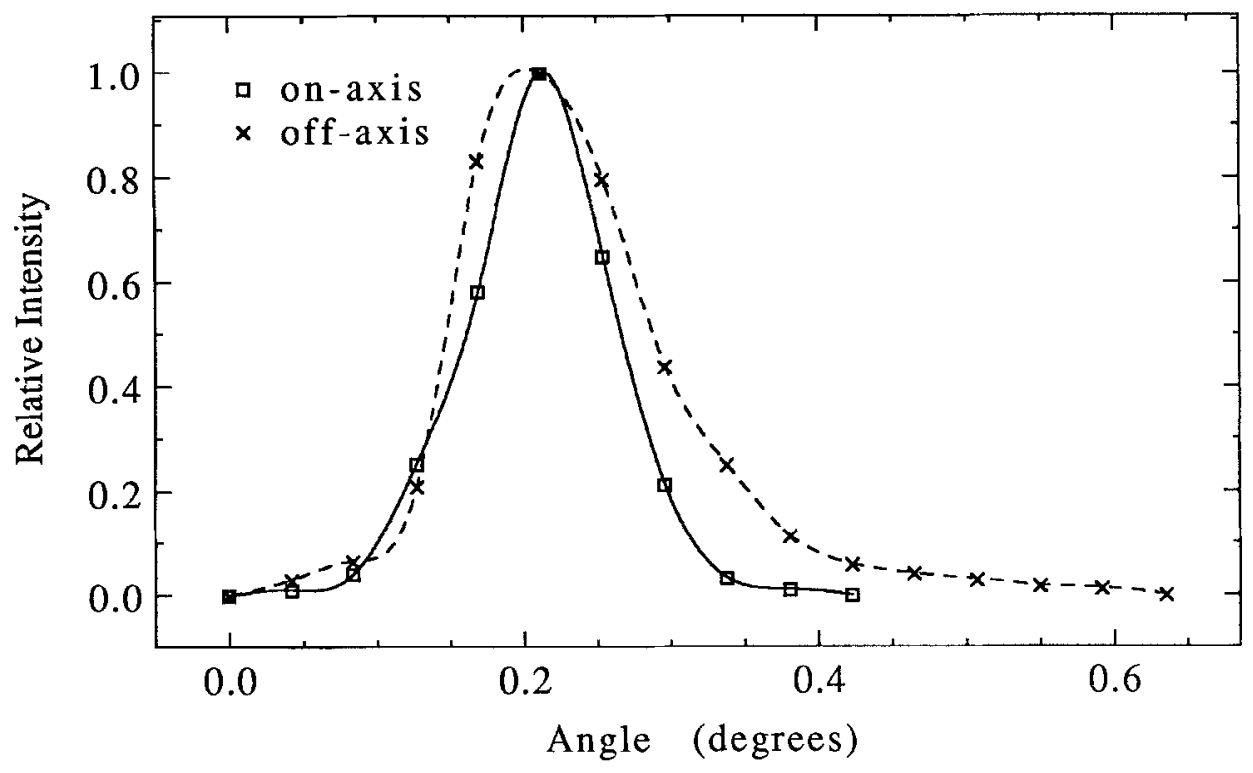

Figure 2. Point spread functions of the $10 \mathrm{~m}$ reflector for the center of the field of view and for a position $1.25^{\circ}$ from the center.

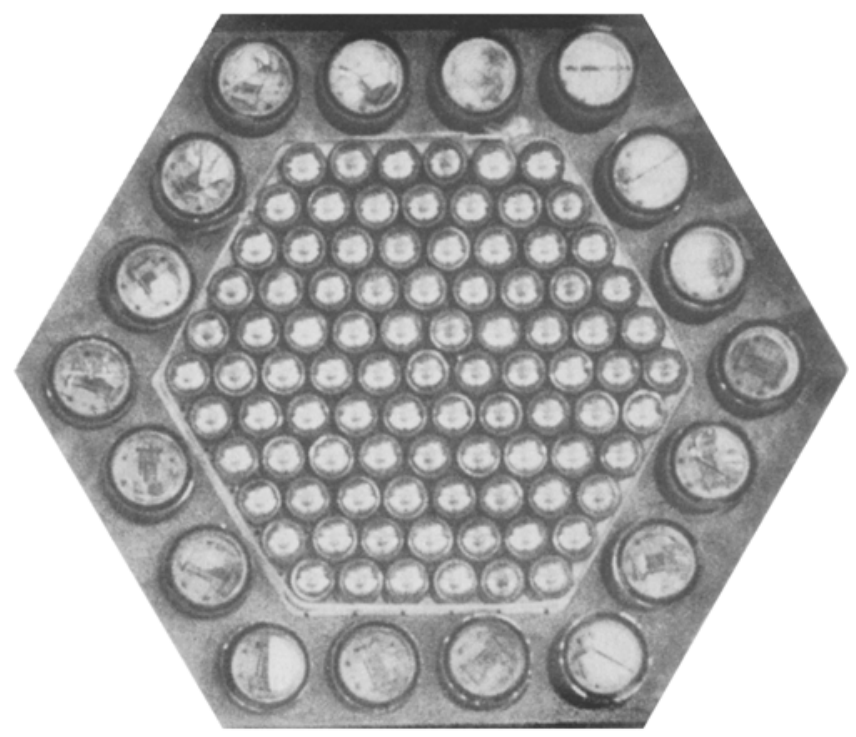

Figure 3. Arrangement of the 109 photomultiplier tubes in the focal plane of the $10 \mathrm{~m}$ reflector. 
sky noise into the PMT channel as their light crosses the tube face. Under such circumstances, the tube voltage might be turned off, effectively removing the tube from the camera. The second purpose is to provide a crude, but simple, check on the pointing of the telescope using bright stars. The tubes are placed on a $0.25^{\circ}$ matrix, and light from a star falls essentially onto a single tube but with a weak halo which is measurable by the neighboring tubes. Consequently the pointing can be checked with an accuracy of approximately $0.1^{\circ}$.

\section{Data Acquisition Electronics}

The data acquisition electronics measures and records a 109-pixel Cherenkov-light image and an arrival time for each event. Since radio pulsars and binary $\mathrm{x}$-ray pulsars are among the classes of objects frequently observed, it is important to measure times accurately and to have as small a deadtime as is feasible in order to minimize distortion of Fourier spectra for periodic sources. The system used at present has evolved over a period of several years and now consists primarily of standard CAMAC modules; several custom-built devices are used to interface various clocks to CAMAC modules. Data from the CAMAC crate are written onto hard disk by a Digital Equipment Corporation LSI $11 / 73$ computer and later transferred to magnetic tapes. The computer also continuously performs tests of the quality of the incoming data as described below and in section 5. Data are analyzed off-line using computers located at the various collaboration institutions.

A block diagram of the overall system is shown in fig. 4. Several logic standards (ECL, fast NIM, and TTL) are used but converters from one standard to another are not shown in the diagram to avoid unnecessary clutter. Required fanouts are omitted from the figure for the same reason. Signals from PMTs in the focus box of the reflector travel into the telescope control building through RG58 cables, 50 meters long, and then into a bank of amplifiers (Lecroy 612A). Each amplifier channel has two outputs, one of which is connected to an analog-to-digital converter (ADC) as shown in the figure. For the inner 91 tubes, the second output from each amplifier is sent to a CAMAC discriminator which is used in determining Cherekov-light triggers. Since it takes a finite time for the hardware to recognize camera triggers, the PMT pulses sent to the ADCs pass through delay cables 60 nanoseconds long. This centers the pulses within the ADC gate period. The ADC bank consists of 10 CAMAC units (Lecroy 2249A), each accepting up to 12 inputs.

The system can be triggered in several modes. An event for which any $\mathrm{N}$ of the inner 91 tubes produces pulses with amplitude exceeding about 40 photoelectrons per tube initiates a standard trigger. The minimum multiplicity, $N$ (normally 2) required for this mode can be set to any value, and the threshold value of 40 photoelectrons is also adjustable. The hardware which accomplishes this trigger mode is as follows. The amplifier outputs for the inner 91 channels are connected into a bank of 6 CAMAC discriminators (LeCroy 4413), each of which accepts up to 16 inputs. Each of the discriminators has a high impedance analog summing output which produces a pulse whose height is proportional to the number of channels exceeding threshold. The analog outputs of the 6 discriminators are daisy-chained together and the summed signal is thus proportional to the total number $(N)$ of channel discriminators which have fired. This summed signal is introduced into a "trigger discriminator" which fires and causes the ADCs to be gated for events with sufficiently high multiplicity. 


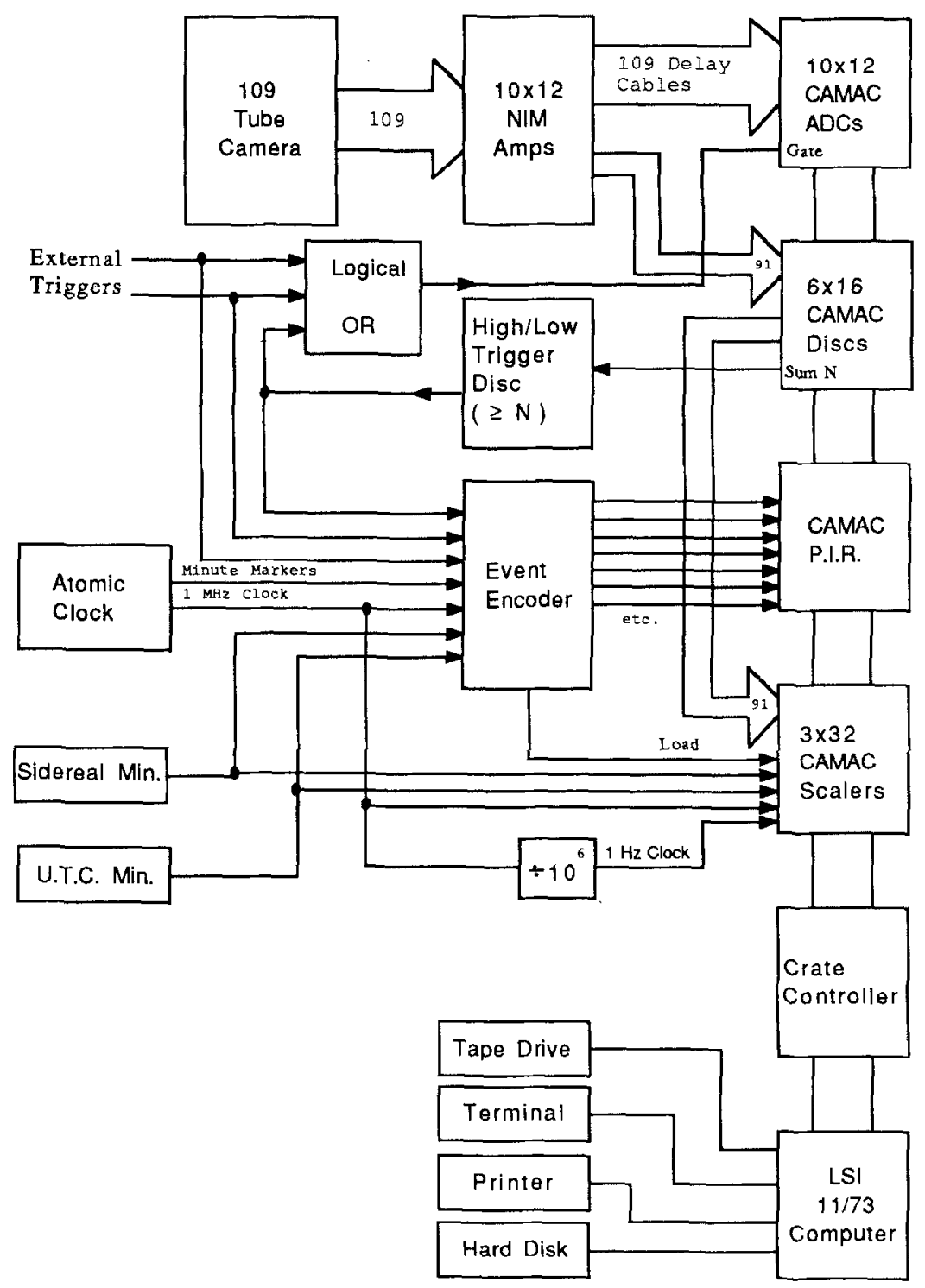

Figure 4. Block diagram of the data acquisition electronics for the imaging detector. 
Unfortunately, the summed signal rises more quickly for large multiplicities. If a normal discriminator were used to generate the trigger when $\mathrm{N}$ is exceeded, it would fire sooner for higher multiplicities and the gate pulse would also arrive sooner at the bank of ADCs. This correlation between the multiplicity and the arrival time of pulses at the ADCs would require uncomfortably long $\mathrm{ADC}$ gate times to insure that all the charge was collecled. The problem is solved by using a high/low pair of discriminators for the multiplicity trigger. One of the discriminators is set to fire at a very low level (therefore frequently) with an output that is gated by the second discriminator with a higher threshold which sets the multiplicity. Thus timing of the ADC gate pulse is set by the "low" trigger discriminator whereas multiplicity is set by the "high" trigger discriminator.

The ADCs are gated for 25 nanoseconds. This relatively long gate period is required because of (1) widening of PMT pulses by the long journey from the telescope focal plane through RG58 cable, (2) variations in PMT transit times, and (3) an intrinsic 6 nanosecond spread in arrival times due to the unusual design of the 10 meter reflector. As described in section 6 the system can be triggered externally in other modes which also cause the ADCs to be gated and digitized. The relative arrival times of events are measured by counting a $1 \mathrm{MHz}$ clock and a $1 \mathrm{~Hz}$ clock with latching CAMAC scalers (LeCruy 4434). These scalers run continuously, and, when an event is recognized by the trigger hardware generating a load signal, the current scaler value is latched into an internal register which can then be read in a more leisurely fashion by the computer through the CAMAC crate controller. Except for a 220 nanosecond deadtime following the load signal, the scalers continue to count. The scalers have 24 bit resolution which implies that the $1 \mathrm{MHz}$ scaler overflows every 16.777216 seconds. The purpose of the $1 \mathrm{~Hz}$ scaler is simply to keep track of overflows in the $1 \mathrm{MHz}$ clock. The $1 \mathrm{MHz}$ clock signal comes directly from a portable atomic clock and is also divided by $10^{6}$ to provide a synchronous $1 \mathrm{~Hz}$ signal.

The bank of scalers consists of 3 units, each with 32 inputs, giving a total of 96 channels. Two channels are used for recording the $1 \mathrm{MHz}$ and $1 \mathrm{~Hz}$ clocks just described, and 91 are used to count the number of times that each of the discriminators for the inner trigger tubes has fired. This makes it possible for the computer to monitor and display the frequency with which each channel discriminator fires ( i.e., the "activity" of the channel) in order to locate possible noise sources and channels with abnormally high or low gains.

Timing events are also injected directly into the data stream. These are generated by three separate clocks: (1) a U.T.C. clock (True Time Division 60-DC) based on WWVB which produces events every U.T.C. minute with an accuracy of about 0.5 millisecond, (2) a portable sidereal clock (Sulzer) which injects events every sidereal minute, and (3) a portable Rubidium clock which produces events separated by 1 minute intervals but shifted by 30 seconds to avoid collisions with U.T.C events. The portable atomic clock is calibrated monthly when it is physically taken to Fort Huachuca, Arizona, 100 miles away where absolute time is maintained to better than 1 microsecond. The U.T.C. clock markers are normally used to fix the absolute times of events to about 0.5 millisecond, although, if necessary, the alomic cluck markers can be used to improve absolute time estimates. Injecting timing events directly into the data stream removes many possible systematic errors and the use of three independent clocks, although redundant, makes it easier to check for any remaining systematic effects. Two of the scaler channels are also used to record the total 
number of sidereal and U.T.C. events for consistency checks.

Recognition of the type of event ( $N$ out of 91 trigger, timing events, etc.) is accomplished by a custom-built "Event Encoder." Each event is given an "event code" ranging from 1 to 12 by the Event Encoder, and a single logic pulse is released on one of twelve output lines. These lines are connected to a Jorway Model 63 Priority Interrupt Register (P.I.R.) which stores the event code (corresponding to the line which carried the pulse) and generates a LAM interrupt. The computer then reads the event code and the two scalers counting $1-\mathrm{Hz}$ and $1-\mathrm{MHz}$ timing pulses. If the event is a genuine Cherenkov trigger or a calibration event (section 6) the 109 $\mathrm{ADC}$ values are recorded along with the event time; if the event is an injected timing marker, the 91 values of the "activity" scalers and the sidereal and U.T.C scalers are recorded instead.

The Event Encoder also generates the "load" signal that causes the scalers to latch their current values into internal registers to be read later by the computer. Although the latching deadtime of the scalers is small (220 nanoseconds) it is important that 1 -second and 1-microsecond pulses do not arrive during this dead period or else a systematic bias is introduced into the time scale. This problem, due to events occuring asynchronously with respect to the $1-\mathrm{Hz}$ and $1-\mathrm{MHz}$ clock pulses, is eliminated in the following way. When a trigger-event or timing-event signal arrives at the Event Encoder, the device waits until the next pulse arrives from the 1-MHz clock, and generates a scaler-load signal on the falling edge of the pulse. This ensures that $1-\mathrm{MHz}$ clock pulses cannot arrive at the scaler during the 220 nanosecond dead period. Since the $1-\mathrm{Hz}$ clock is derived from, and synchronous with, the $1-\mathrm{MHz}$ clock, it is also impossible for the $1-\mathrm{Hz}$ scaler pulses to be missed because of latching deadtime.

\section{Software for Data Acquisition and Control}

The data acquisition and control system for the camera is illustrated schematically in fig. 5. An Apple II microcomputer, operating under a FORTH environment, is used to control the motion of the telescope. Synchro-to-digital shaft encoders are used to provide positional feedback, with a resolution of $0.04^{\circ}$. The absolute position is also monitored using an intensified video camera mounted on the $10 \mathrm{~m}$ reflector. Displays associated with both the tracking system and the video camera are recorded at regular intervals on video tape for purposes of verification during subsequent data analysis. The quality of the star field images on the video also provide a check of sky clarity in the source direction. An additional check on overall sky quality is provided by a photomultiplier tube continuously monitoring Polaris. The current level, fed to a chart recorder, gives a good indication of the presence of even thin cirrus cloud which may otherwise remain undetected.

As described in the previous section, the data is acquired via an LSI 11/73 Digital Equipment Corporation computer interfaced to the CAMAC crate. In addition to reading in the data and storing it on the internal hard disk, this computer uses a customised software package written in FORTRAN and assembly language to perform a number of on-line monitoring tasks. Before the start of each data run, the operator supplies the system with identifying information (source name, coordinates, starting time, etc.) which is stored as a data block at the start of the relevant filc. During the course of the run, the system permits the operator to interactively monitor several 

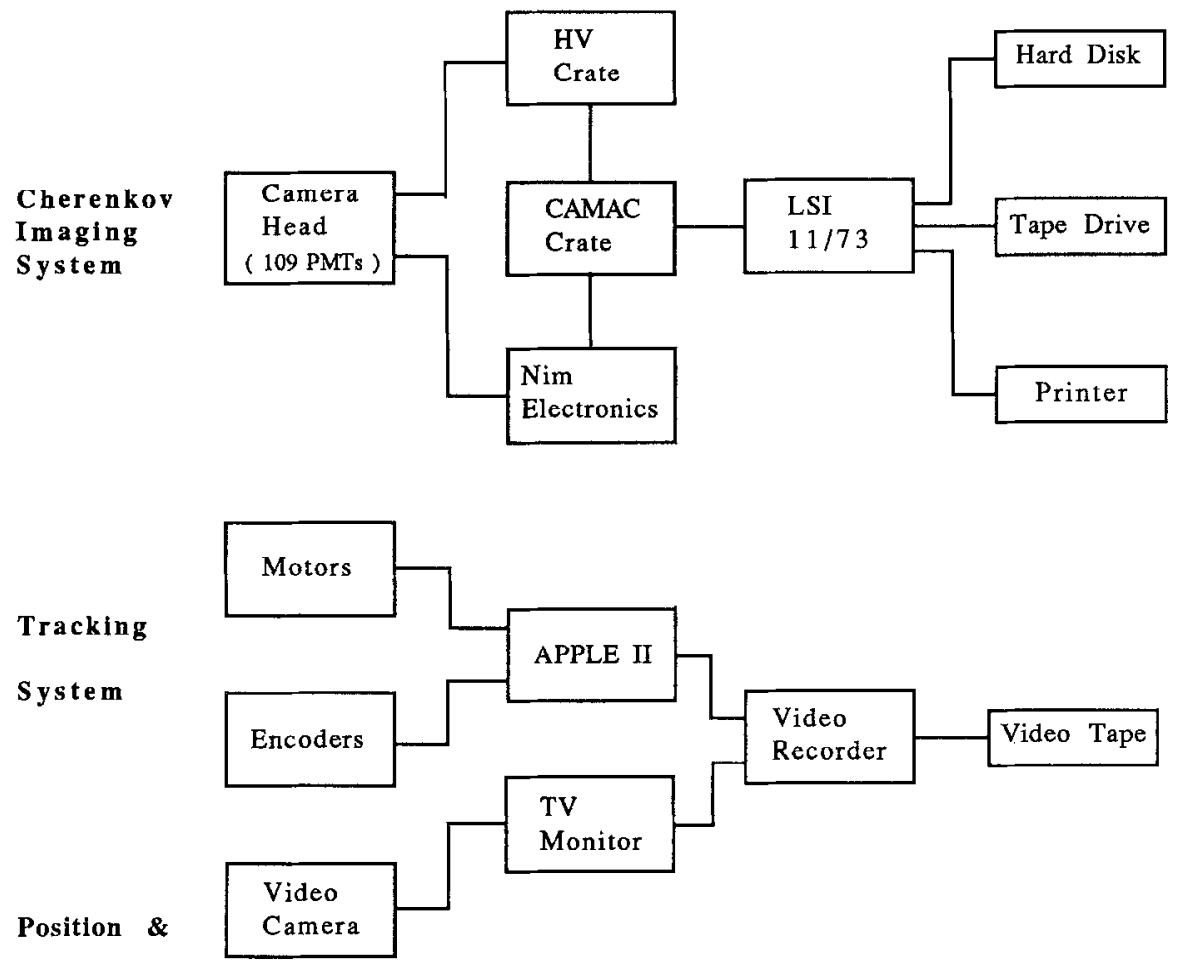

Sky Quality

Verification

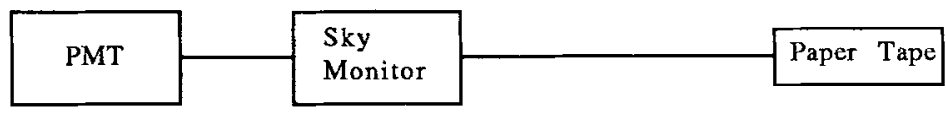

Figure 5. Block diagram of the various elements in the control system associated with the Cherenkov Imaging telescope. 
aspects of the system performance:

i) individual events: the $\mathrm{ADC}$ outputs are displayed in the hexagonal image pattern on the display monitor,

ii) PMT singles rates in histogram form for the previous sidereal minute; this reveals if any tubes are triggering at an excessive rate,

iii) PMT singles rates and coincidence rate since start of run; provides an on-line monitor of system stability and sky quality (this display is updated each sidereal minute and is also sent to a printer),

iv) PMT pulse height spectra using accumulated events; indicates if relative gains of tubes are reasonably balanced,

v) present status of run: elapsed time, number of events accumulated, and amount of free space remaining on hard disk.

In addition to selecting any of these monitoring modes, the operator has the facility of entering comments pertinent to sky quality, system performance, etc. These comments are stored in a reserved data block at the head of the file along with the run identification information.

Each event is recorded as 128 16-bit words. Four words are used to store relative timing information to microsecond accuracy, and a further two words are used to count the UT and sidereal minute markers. One word is reserved for a code which uniquely identifies the nature of each event (eg. Cherenkov event, UT timing event, etc.) and one word is used to store a run identification code. The remaining 120 words are used to store the ADC values (109 tubes in the main camera head, and 11 spare channels). One sidereal and two UT timing events are recorded at minute intervals; in the sidereal events, the words normally reserved for the ADC values are used to record the cumulative singles rates (inner 91 PMTs only). Events are transferred from the CAMAC crate by direct memory access and are buffered in core memory to reduce disk access time, thereby decreasing the average system deadtime to 20 msec. Since typical rates are 4 to 5 events per second, deadtime corrections are small. A partition on the 300 Mbyte hard disk is reserved for data. At the end of a night's observing, the data in this partition is transferred to 1600 bpi magnetic tape. Multiple copies of the data are subsequently made using a more efficient blocking system on an Ultrix-based Microvax system for distribution to the various analysis centers.

Separate programs are available on the LSI $11 / 73$ for use during system testing and calibration. One such program interfaces directly with the high voltage crates and adjusts the voltages applied to the tubes depending on their response to signals from the fast nitrogen flashlamp (see next section). Voltages are not, however, adjusted during the course of an observing run.

\section{System Performance}

The high resolution camera is triggered on the basis of a hardware majority logic decision: a threshold of 40 photoelectrons is set in each channel, and an event is registered if any $N$ of the inner 91 channels exceeds this threshold, where $N$ can be varied from 1 to 6 . Both the individual thresholds and the value of $\mathrm{N}$ determine the overall threshold energy and collection area of the detector. For the 2 out of 91 trigger used in practice, simulations indicatc a threshold cncrgy of $0.3 \mathrm{TeV}$ with a collection area for gamma rays of $3 \times 10^{8} \mathrm{~cm}^{2}$ (with a factor of 1.5 uncertainty in both 
estimates). Fig. 6 shows the 2 out of 91 trigger rate as a function of zenith angle. The fitted curve is of the form $\operatorname{Cos}^{n} \mathrm{z}$, where $\mathrm{z}$ is the zenith angle. A slow decline in rate with increasing $\mathrm{z}$ is found, with a value of $\mathrm{n}$ of 0.9 . This may be compared with a value for $\mathrm{n}$ of 2.0 found by Rieke (1969) using a single $12.5 \mathrm{~cm}$ PMT at the focus of the $10 \mathrm{~m}$ reflector. The difference is due in large part to the ability of the high resolution system to trigger on narrow images; as the zenith angle is increased, showers of a given energy will reach maximum development further from the detector and will thus subtend smaller angles. Such showers will more readily trigger a narrow-angle detector, giving rise to a slower decline in rate. This narrowing of the angular extent of the images is evident in fig. 7 which shows the ratio of the Cherenkov light detected by the two most strongly illuminated PMTs to the total light striking the camera as a function of zenith angle. The ratio increases from under $30 \%$ at $\mathrm{z}=0^{\circ}$ to over $50 \%$ at $\mathrm{z}=70^{\circ}$.

To fully quantify the true Cherenkov trigger rate, account must be taken of other possible trigger sources. The main source of contamination, particularly at large zenith angles, is due to charged particles generating Cherenkov light within the glass of the PMTs. At large values of $\mathrm{z}$, a near vertical particle sees a larger cross-section of glass, and also passes through two or more tubes more readily. The trigger rate due to such events was measured by placing a light tight lid over the camera. This single-particle rate is shown in fig. 8 ; it contributes $5 \%$ of the total rate at $z=0^{\circ}$, increasing to over $20 \%$ at $\mathrm{z}>60^{\circ}$. In practice, this degree of contamination does not usually prescnt a problem as most of the uscful data on sources is taken at small (< $45^{\circ}$ ) zenith angles where image features are better quantified due to spreading of the light over several pixels. Furthermore, even though these single-particle events have narrow angluar widths, and therefore mimic the appearance of gamma rays, they should not show any preference for on-source or control regions. In principle, such events could be removed entirely by employing an anticoincidence particle shield around the PMTs.

The rate of accidental triggers (due to random fluctuations in the outputs of the PMTs) may be estimated from (Janossy, 1944)

$$
\mathrm{R}=\mathrm{nk}^{\mathrm{n}_{\mathrm{T}}^{\mathrm{n}-1}} \mathrm{~Hz},
$$

where $\mathrm{n}$ is the number of PMTs involved in the coincidence, $\mathrm{k}$ is the singles rate in each tube (assumed the same for all tubes), and $T$ is the duration of the coincidence window (10nsec). In the case of the imaging detector, the number of channels involved in the coincidence ( $\mathrm{n}$ ) is less than the total number of channels in the trigger $(m=91)$, so the above expression is multiplied by the number of possible combinations of the $m$ PMTs that can form an $n$-fold coincidence. This gives an accidental trigger rate of

$$
\mathrm{R}=\frac{\mathrm{m} !}{(\mathrm{m}-\mathrm{n}) !(\mathrm{n}-1) !} \quad \mathrm{k}^{\mathrm{n}} \mathrm{T}^{\mathrm{n}-1} \mathrm{~Hz}
$$

For a two-fold coincidence level, and a typical singles rate of $1 \mathrm{~Hz}, \mathrm{R}$ is $8.2 \times 10^{-5}$ $\mathrm{Hz}$, or 1 random coincidence in 3.4 hours. The contribution of accidental events to the overall trigger rate is thus negligible. 


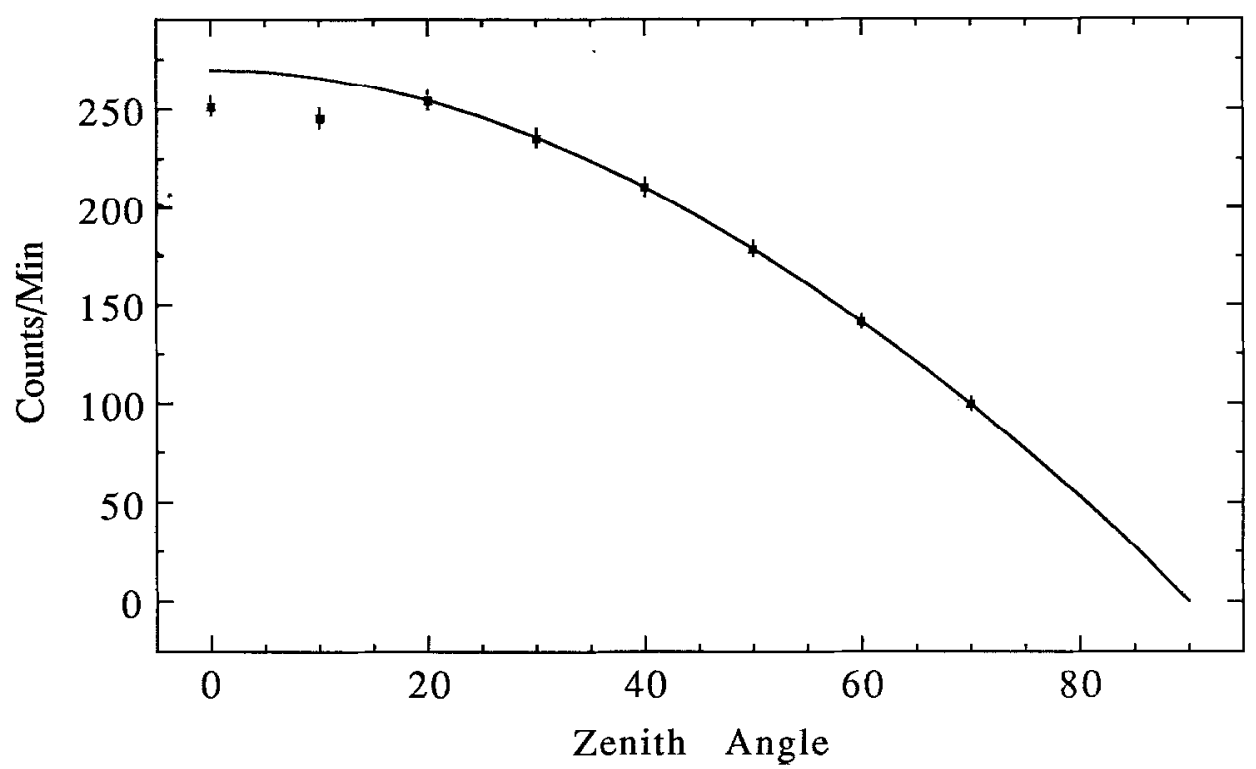

Figure 6. Trigger rate (2 out of 91) as a function of zenith angle. The fitted curve is of the form $\operatorname{Cos}^{n} \theta$ where $\theta$ is the zenith angle and $n$ is 0.9. As is evident here, it is generally found that the count rate flattens somewhat at low zenith angles.

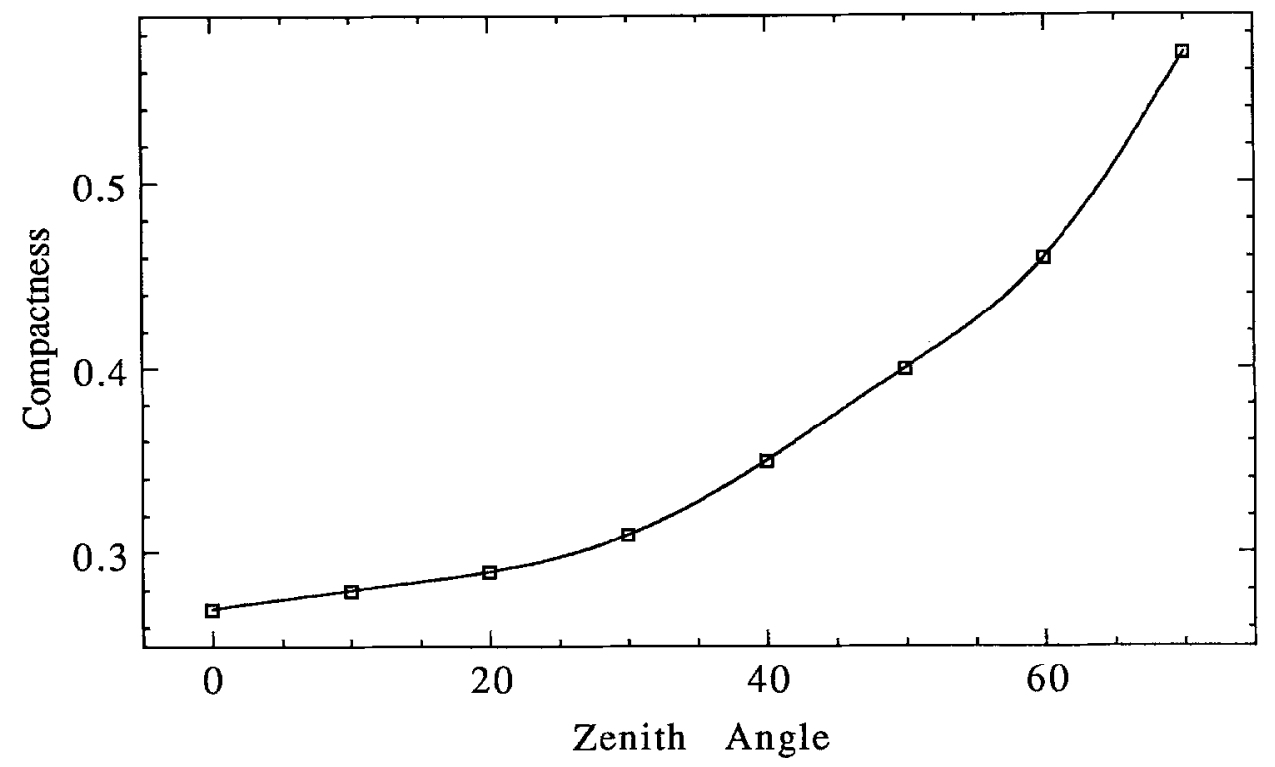

Figure 7. Ratio of the Cherenkov light detected by the two most strongly illuminated PMTs to the total light striking the camera ('compactness') as a function of zenith angle. 


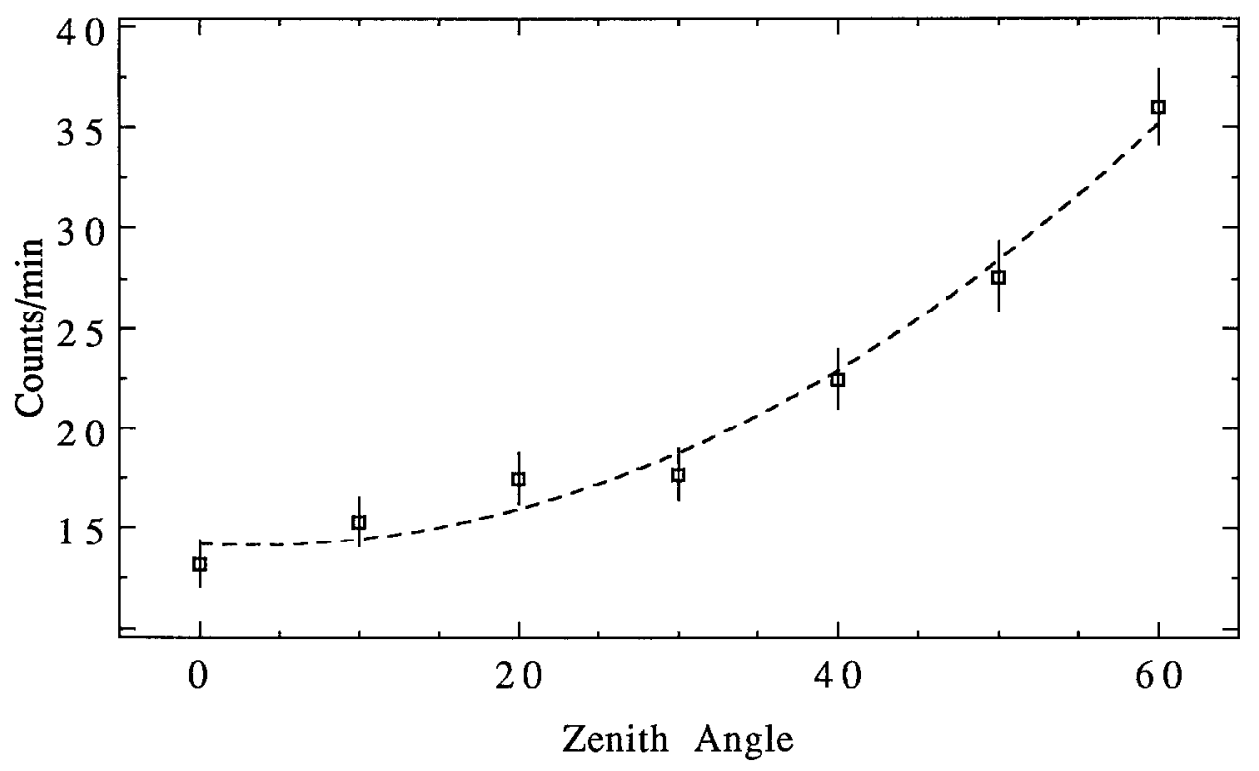

Figure 8. Trigger rate (2 out of 91) due to local cosmic rays generating Cherenkov light within the glass of the PMTs as a function of zenith angle.

\section{Image Calibration and Preparation}

The analysis of each Cherenkov image can best be described as a two-stage process: i) preparation of the image, and ii) pattern recognition. The main task in image preparation is gain normalization ("flat-fielding"). Several special data files are taken at the start and end of each night of observations to assist in this process. The system is triggered internally using a fast oscillator to record the residual 'pedestals' associated with the ADCs (the PMTs are exposed to the night sky but the high voltages are switched off). These pedestals are deliberately set at around 20 digital counts rather than at zero so that negative fluctuations may be recorded (such as may occur due to fluctuations in the AC-coupled current from the night sky light). The mean pedestal value for each tube is calculated and subtracted from every image. A second pedestal file is recorded with the high voltages switched on and with the camera exposed to the night sky in order to gain information on the role of night sky fluctuations - such files are used to characterize the noise in the system.

To aid in the flat-fielding procedure, a file of approximately one thousand images is recorded using a fast nitrogen flash lamp (Optitron) shining directly on the focal plane. Because of the spectral differences between the flash lamp and the Cherenkov light, and due to relative spectral differences among the PMT cluster, this calibration file is used only to find the relative gains of the rings or 'zones' of PMTs. To find the relative gains within a zone, the aggregate Cherenkov light from events taken over 
several hours is used. Given the azimuthal symmetry within a zone, it is assumed that the pulse height spectra of each tube would be similar if their relative gains were similar. Thus, integral pulse height spectral responses to Cherenkov images are compared to determine appropriate relative normalization factors within each zone, and the flash lamp data are used to apply an additional intra-zone correction. The resulting 109 mulliplicative gain factors are applied to each Cherenkov image after pedestal subtraction.

A series of software filters is then applied to the resulting normalized images to reduce as much as possible the effects of noise fluctuations in the tubes. This noise arises from several sources: i) night sky light fluctuations, ii) electronic noise, and iii) signal noise (Lewis et al. 1987). Night sky light is recorded together with Cherenkov light in the $25 \mathrm{nsec}$ integration period. The magnitude of the fluctuation on this background light can be gauged by artificially triggering the system with the high voltage applied to the tubes and determining the standard deviations on the ADC pedestals. For a typical source region, this is found to be 3.0 digital counts for the $2.9 \mathrm{~cm}$ tubes. The electronic noise may be quantified by measuring the fluctuations in the ADC pedestals with no voltage applied to the PMTs. This is found to be 0.8 digital counts; we can therefore neglect the electronic noise in most cases as other noise sources dominate. The measured digital counts for a PMT gated into the ADC is

$$
\mathrm{M}_{\mathrm{i}}=\mathrm{S}_{\mathrm{i}}+\mathrm{B}_{\mathrm{i}},
$$

where $M_{i}$ is the measured count in tube $i, S_{i}$ is the count due to the actual signal, and $\mathrm{B}_{\mathrm{i}}$ is the count due to the background sky light. $\mathrm{As} S$ and $\mathrm{B}$ are uncorrelated, the variance on $M$ is the sum of the variances on $S$ and $B$. Experimental determination of the variance of $\mathbf{B}$ was described above. The variance of $\mathbf{M}$ for each tube $\mathrm{i}$ was determined by measuring the variance of the signals using the nitrogen flash lamp. An average for all 91 of the $2.9 \mathrm{~cm}$ tubes gives the empirical result

$$
\sigma^{2} \mathrm{M}=1.35(\mathrm{~S})+(3.0)^{2}, \mathrm{~S} \text { in digital counts. }
$$

The minimum possible variance of $\mathbf{M}$ is set by shot noise in the number of photoeletrons produced; this minimum variance is equal to the mean in terms of photoelectrons. The avcragc overall gain of the camcra was determincd in the laboratory by directly measuring the current gain of each tube and the gain of the processing electronics, giving an average factor of $1.0 \pm 0.2$ digital counts per photoelectron. The noise in $S$ therefore exceeds the photoelectron shot noise (Poisson) limit by a factor of about 1.35. This is reasonable in view of noise in subsequent amplification by the dynode chain. Essentially all photomultiplier dynode secondary-electron distributions are intermediate between relatively quiet Poisson and relatively noisy exponential limits (Engstrom, 1980), and the value of 1.35 falls between values calculated using these limiting distributions. From the expression for the overall variance given above, the resulting noise level may be used to i) optimally filter the image prior to image analysis, and ii) assess the errors in the pattern classification arising from noise.

At present, simple filter processes based on Monte Carlo simulations are applied to reduce the level of distortion of the Cherenkov image due to the noise contributions discussed above. Prior to any attempts at image analysis, the following filtering is 
(i) Recorded pixel values in digital counts

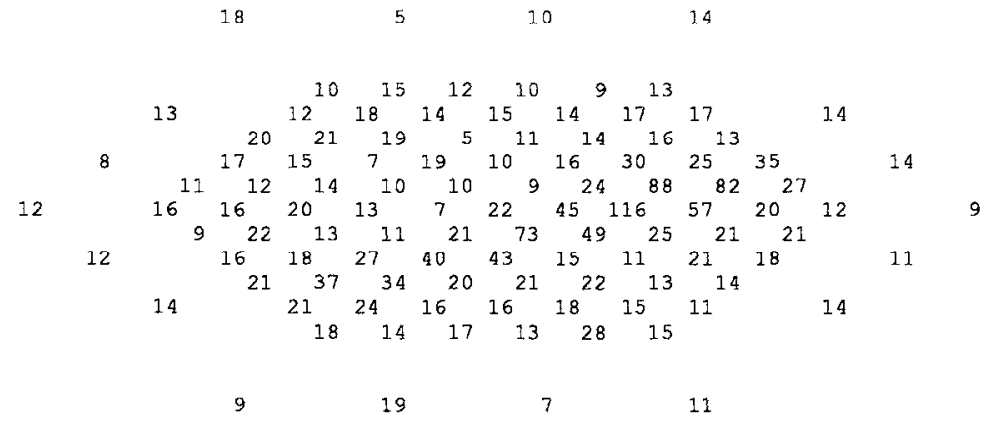

(11) After calibration (pedestal subtraction and flat-fielaing)

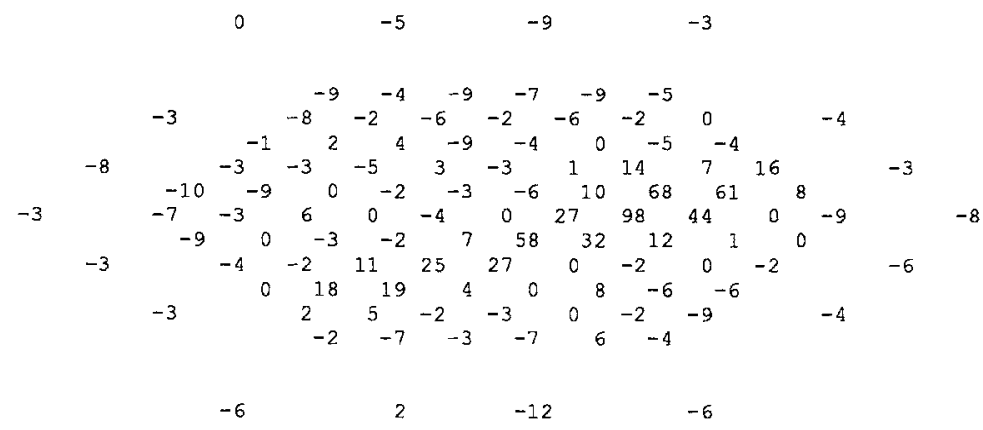

(iii) After noise reduction

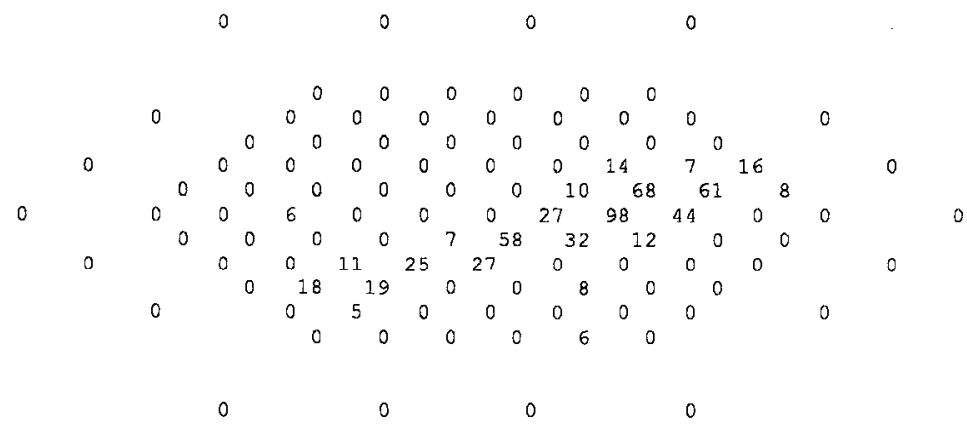

Figure 9a. A typical image of a Cherenkov flash associated with a cosmic ray event indicating the effects of the various stages of calibration and filtering. The values of the image features for this event are: Length = $0.51^{\circ}$, Width $=0.17^{\circ}$, Miss $=0.27^{\circ}$, Distance $=0.38^{\circ}$, Azwidth $=0.38^{\circ}$, Compactness $=0.28$, Zone $=2$ (the innermost tube is designated as zone 0 ). This image would be classified as a background cosmic ray event due to the large values of Length, Miss, and Azwidth. 
(i) Recorded pixel values in digital counts

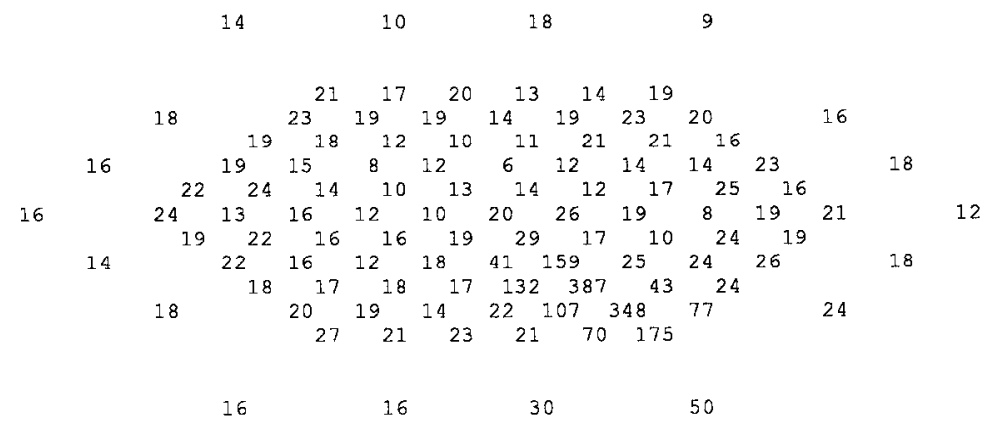

(ii) After calibration (pedestal subtraction and flat-fielding)

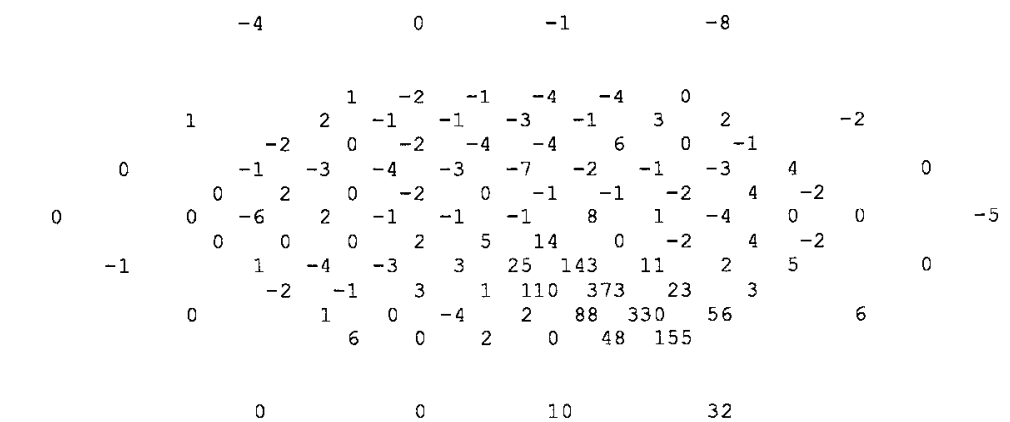

(iii) After noise reduction

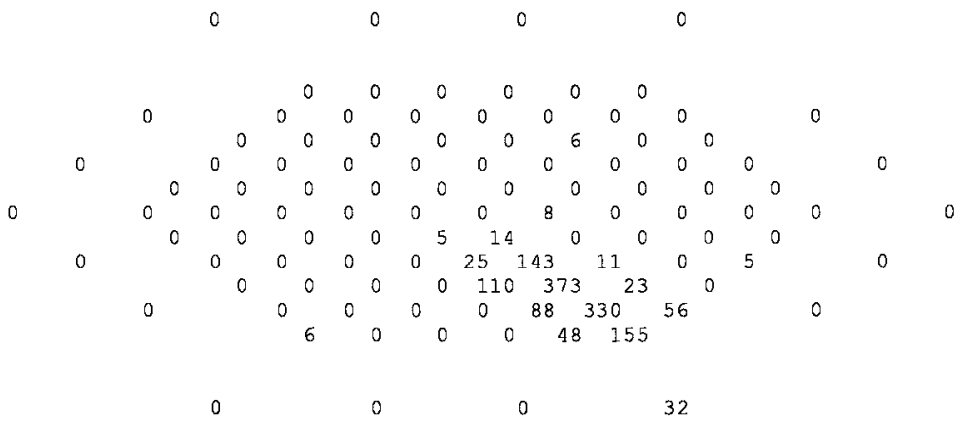

Figure 9b. An image which would be accepted as a candidate gamma-ray event based on its image feature values, which are: Length $=0.29{ }^{\circ}$, Width $=0.14^{\circ}$, Miss $=0.07^{\circ}$, Distance $=0.86^{\circ}$, Azwidth $=0.14^{\circ}$, Compactness $=$ 0.49, Zone $=3$. 
done after pedestal subtraction and normalization:

i) a software trigger is applied, demanding at least 40 digital counts (equivalent to about 40 photoelectrons) in at least 2 of the 91 triggering pixels,

ii) any pixel with less than 5 digital counts is set to zero,

iii) any pixel with less than $0.5 \%$ of the total light (summed over all tubes) is set to zero,

iv) all pixels more than $1.4^{\circ}$ from the maximum pixel signal are set to zero.

Typical images of cosmic ray and candidate gamma-ray events are shown in fig.

9, indicating the effects of the various stages of image calibration and filtering, prior to application of pattern recognition algorithms.

\section{Pattem Recognition}

In this, the most critical stage of the data analysis, the ultimate aim is to optimally enhance the signal to noise ratio using the recorded image information. This is essentially a problem in pattern recognition: simulations suggest the characteristics of both gamma-ray and proton images, and this information may be used to classify images in the real data. Furthermore, even in the absence of the simulated images, the class of background images may be characterized using data taken on control regions, thus allowing for the possibility of isolating an image class which differs both from hackground and the simulated class of gamma rays.

There exist many different approaches to pattern recognition, dividing into two main categories: i) the discriminant approach and ii) the structural approach. In the discriminant approach, a set of characteristic measurements, or 'features', are extracted from the patterns. Each pattern is represented by a vector in n-dimensional feature space, and the task of the recognition algorithm is to partition this feature space. In the structural approach, each pattern is expressed as a composition of its component subpatterns or 'pattern primitives'. The pattern is then described in terms of the set of pattern primitives and their compositional relationships. The recognition algorithm may test for the occurrence of certain primitives or for a particular relationship between primitives. The method of template-matching falls into this category: the set of primitives and compositional relationships of the test image are compared with those of the reference pattern and a measure of similarity is determined.

For the analysis of Cherenkov images, we have concentrated on the discriminant approach. The initial task is to transform from pattern space (with 109 dimensions in our case) to a suitable feature space. There are several desirable properties associated with an optimal feature space (Andrews, 1972): i) low dimensionality, ii) good clustering within classes, and iii) large separation between classes. All these properties favor simpler and more robust decision boundaries separating the different classes of points in feature space. Examination of the simulated images from both the proton and gamma classes indicates that the main discriminating features are associated with angular extent and orientation relative to the center of the field of view. Differences in the angular extent arise from the different compositions of the cascades which in turn leads to different rates and depths of development in the atmosphere. Differences in orientation arise from the fact that all the signal showers develop parallel to the axis of the detector (assuming that the source is located at the center of the field of view) whereas the background shower directions are randomly distributed. Other features are also extracted from each image, eg. shower size (sum of all the ADC 
values) and position in the focal plane of the largest ADC value. Such features may contribute to the discrimination process depending on the source spectrum.

A seven-dimensional feature space was tested by Hillas (1985) on simulated proton and gamma-ray images; five of these features are illustrated in fig. 10. 'Length' and 'Width' are the rms spread of light in directions parallel and perpendicular to the image axis (the line which minimises the signal-weighted sum of squares of

perpendicular angular distances of pixels). 'Compactness' is a feature which expresses the degree of light concentration - it is simply the ratio of the sum of the two largest ADC values to the total. 'Miss' is the perpendicular distance of the center of the field from the image axis - a small 'miss' value indicates good alignment of the shower axis with the source direction. 'Azwidth' is a feature which combines both angular extent and orientation - it is the rms image width along a new axis which joins the center of the field to the centroid of the image. 'Distance' is the angular distance of the image centroid from the center of the field. Finally, Hillas (1985) also classified the images in terms of the location of the maximum pixel signal. The array was divided into rings or 'zones' for this purpose. This was done as image shape for a given shower varies as a function of the shower impact parameter on the ground relative to the location of the telescope.

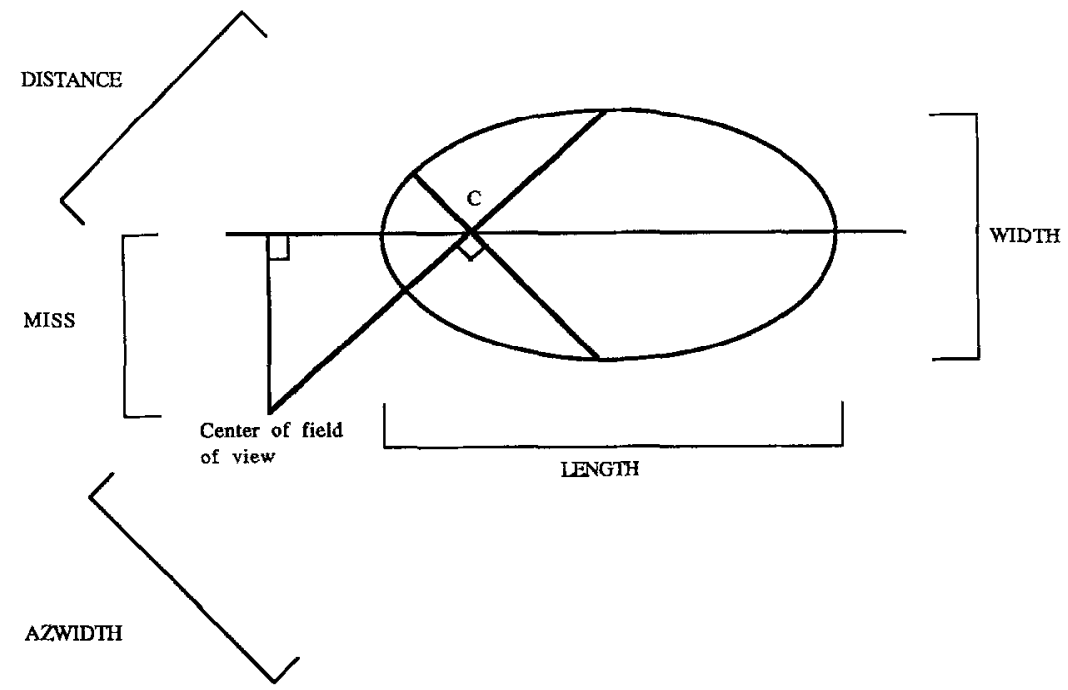

Figure 10. The features used to classify the Cherenkov images.

$C=$ Centroid of image.

The discrimination efficiency may be quantified by the ratio

$$
Q=\left[\frac{N \gamma}{N \gamma_{0}}\right] /\left[\frac{N p}{N p_{0}}\right]^{\frac{1}{2}},
$$

where $\mathrm{N}_{\gamma_{0}}$ and $\mathrm{N}_{\mathrm{po}}$ are the total number of gamma-ray and proton images, and $\mathrm{N}_{\gamma}$ 
and $N_{p}$ are the numbers surviving the discrimination process. This $Q$ ratio is effectively the factor by which the significance (expressed in standard deviations) of the signal to noise ratio is improved. Hillas (1985) first determined the decision boundaries which optimised $Q$ for the individual features, and then demanded that a particular image lie within a region defined by a majority (eg. 4 out of 6 ) of these boundaries. Thus, a gamma-ray image may in principle lie anywhere in the feature space, but there are certain regions where it will be found with higher probability. For the original 37-pixel camera, Hillas (1985) predicted $Q$ values in the range 4 to 7 (60\% to $70 \%$ of the gamma-rays retained, with $98 \%$ to $99 \%$ of the protons rejected). A similar analysis for the 109-pixel camera predicts an improvement in these $Q$ values by a factor of approximately 2 - ie. a $Q$ value of 10 should be attainable with the high resolution camera using the seven-dimensional feature space discussed above.

Additional image features have been proposed by other workers on the basis of independent sets of simulations. In addition to the features described previously, Plyasheshnikov and Bignami (1985) tested the discrimination efficiency of features based on a 'guard-ring' approach (ratio of light within a radius of $0.3^{\circ}$ of the center of the field to light in an annulus of radius $1^{\circ}$ surrounding the central region) and on the degree of fluctuation in the distribution of light along the image axis (it was found that the simulated proton images exhibited a larger degree of fluctuation). Zyskin (1989) has proposed a feature called 'prolength', the projection of the image axis onto the line joining the centroid with the center of the field. This is similar to 'azwidth' in that it combines both angular extent and orientation, but it is claimed on the basis of independent simulations to give better discrimination.

The first successful application of the Cherenkov imaging technique in VHE gamma-ray astronomy in which the Crab Nebula was detected at a significance level of 9 standard deviations (Weekes et al. 1989) was based on the simplest type of discrimination: a partition in a one-dimensional feature space ('Azwidth'). Using the original 37-pixel camera, a $2 \sigma$ effect in uncut data became $9 \sigma$ after imaging, indicating a $Q$ factor of order 4.5 (with large uncertainty due to the uncertainty in the uncut excess). The effect was reproduced with the 109-pixel camera (Lang et al. 1989), and there are preliminary indications that the high resolution camera is of order 2 to 3 times more sensitive than the 37-pixel camera. To date, the imaging technique has not enhanced the significance of signals from sources other than the Crab Nebula, even in situations where tentative signals (at the $99 \%$ confidence level) have been observed in the raw, unimaged data (eg. Reynolds et al. 1990).

The application of pattern recognition techniques is still at a primitive stage in Cherenkov imaging, and it is to be expected that additional gains in sensitivity will be made as more sophisticated discrimination techniques are applied. Apart from defining an optimal feature space, it is most important to devise optimal discrimination decisions which will lead to high $Q$ ratios over wide feature ranges (ie. 'robust' cuts, insensitive to small changes in the distributions of feature values due to different sky conditions, zenith angles, etc.). To date, some of the standard techniques of multivariate analysis such as linear discriminant analysis, principal component analysis, and cluster analysis, have been applied to simulated data with promising results (Zyskin and Komienko, 1989; Komienko, 1989). An additional factor of two in sensitivity gained by such methods could lead to an overall $Q$ value in excess of 20 for the 109-pixel camera compared to a non-imaging detector. Further improvements in sensitivity may be achieved through the use of multiple imaging systems such as the proposed GRANITE detector (Akerlof et al. 1989). 


\section{Acknowledgements}

This work is supported by the U.S. Department of Energy, the Smithsonian Scholarly Studies Fund, and the National Board of Science and Technology of Ireland. A.M.H. and T.C.W. acknowledge the support of a NATO grant.

\section{References}

Akerlof, C.W., Cawley, M.F., Fegan, D.J., Hillas, A.M., Lamb, R.C., Lewis, D.A., Meyer, D.I., and Weekes, T.C. (1989) Proc. Workshop on VHE gamma-ray and neutrino astronomy (Arkansas), to be published in Nuclear Physics B.

Andrews, H.C., (1972) 'Introduction to Mathematical Techniques in Pattern Recognition', Wiley, New York.

Cawley, M.F., Fegan, D.J., Gibbs, K., Gorham, P.W., Hillas, A.M., Lamb, R.C., Liebing, D.F., MacKeown, P.K., Porter, N.A., Stenger, V.J., and Weekes, T.C. (1985) Proc. 19th Int. Cosmic Ray Conf. (La Jolla), 1, 131.

Davies, J.M., and Cotton, E.S. (1957) Journal of Solar Energy, 1, No. 2 and 3, 16-22.

Engstrom, R.W. (1980) 'RCA Photomultiplier Handbook', Appendix G, p160.

Fegan, D.J., McLaughlin, D., Clear, J., Cawley, M.F., and Porter, N.A. (1983) Nucl. Inst. and Methods, 211, 179.

Grindlay, J.E. (1971) Smithsonian Astrophysical Observatory, SP-334.

Hillas, A.M. (1985) Proc. 19th Int. Cosmic Ray Conf. (La Jolla), 3, 445.

Janossy, L. (1944) Nature, 153, 165.

Kornienko, A.P. (1989) Proc. Workshop on VHE gamma-ray astronomy (Crimea), p138.

Lang. M.J., Cawley, M.F., Fegan, D.J., Hillas, A.M., Kwok, P., Lamb, R.C., Lewis, D.A., Macomb, D., Reynolds, P.T., Vacanti, G., and Weekes, T.C. (1989) Proc. Workshop on VHE gamma-ray and neutrino astronomy (Arkansas), to be published in Nuclear Physics B.

Lewis, D.A. (1989) submitted to Experimental Astronomy.

Lewis, D.A., Cawley, M.F., Fegan, D.J., Hillas, A.M., Kwok, P.W., Lamb, R.C., Reynolds, P.T., Porter, N.A., and Weekes T.C. (1987) Proc. 20th Int. Cosmic Ray Conf. (Moscow), 2, 360.

Plyasheshnikov, A.V. and Bignami, G.F. (1985) Nuovo Cimento, 8C, 39.

Reynolds, P.T., Cawley, M.F., Fegan, D.J., Hillas, A.M., Kwok, P.W., Lamb, R.C., Lewis, D.A., Macomb, D., Vacanti, G., and Weekes, T.C. (1990) Proc. 21st Int. Cosmic Ray Conf. (Adelaide), OG 4.2-2.

Rieke, G.H. (1969) Smithsonian Astrophysical Observatory, SP-301.

Stepanian, A.A., Fomin, V.P., and Vladimirsky, B.M. (1983) Izv. Krym. Astrofiz. Obs. 66, 234.

Weekes, T.C., Cawley, M.F., Fegan, D.J., Gibbs, K.G., Hillas, A.M., Kwok, P.W., Lamb, R.C., Lewis, D.A., Macomb, D., Porter, N.A., Reynolds, P.T., and Vacanti, G. (1989) Ap. J. 342, 379.

Zyskin, Yu.L. (1989) Proc. Workshop on VHE gamma-ray astronomy (Crimea), p148.

Zyskin, Yu.L., and Komienko, A.P. (1989) Proc. Workshop on VHE gamma-ray astronomy (Crimea), p143. 